\title{
X-ray Phase Contrast Imaging Technique Using Bilens Interferometer.
}

\author{
D. Zverev ${ }^{1}, \underline{\text { I. Snigireva }}{ }^{2, *}$, V. Kohn ${ }^{3}$, S. Kuznetsov ${ }^{4}$, V. Yunkin ${ }^{4}$, and A. Snigirev ${ }^{1}$
}

1. Immanuel Kant Baltic Federal University, Kaliningrad, Russia

2. European Synchrotron Radiation Facility, Grenoble, France

3. Russian Research Centre "Kurchatov Institute", Moscow, Russia

4. Institute of Microelectronics Technology RAS, Chernogolovka, Russia

*irina@esrf.fr

Nowadays, the laser-like properties of the X-ray beam delivered by the third generation synchrotrons allow the development of in-line or paraxial schemes of X-ray interferometry such as the classical double slit Young's experiment or the phase contrast imaging with a grating interferometer [1-2]. Recently, a bilens interferometer consisting of two parallel compound refractive lenses was proposed [3]. Under coherent illumination, the bilens interferometer generates two diffraction limited mutually coherent beams that are focused at some distance. The size of the focal spots is limited by the diffraction limit and can be less than tens of nanometers. When the beams overlap, they generate an interference pattern with a fringe spacing ranging from tens of nanometers to tens of micrometers, depending on the distance to the observation plane. This simple way of creating an interference pattern opens up new opportunities for development of phase contrast imaging techniques.

As in the case of a classical interferometer, one can easily insert a sample into one of the beams, since they are separated (Fig. 1a). The sample leads to a certain phase shift (delay) of the incident beam shifting the interference fringes. The high sensitivity of the interference pattern to the phase distortions of the beam produced by the sample allows to extract comprehensive information about the sample structure.

To reconstruct the phase profile of the object, it is necessary to analyze the shifts of the interference fringes recorded during the scanning of the sample in the direction perpendicular to the optical axis. The bilens interferometer makes possible to study the samples with dimensions smaller or larger than the distance between separated beams. Unlike a small sample, a large object overlaps both beams. Nevertheless, it is easy to reconstruct the absolute values of the phase profile of the sample using the relative shifts of the fringes obtained when the sample is scanned.

We demonstrated this technique using boron fibre as a sample that is transparent for hard X-rays. This sample is a coaxial structure consisting of a boron shell with a diameter of $100 \mu \mathrm{m}$ and a tungsten core with a diameter of $15 \mu \mathrm{m}$. The experiment was carried out at the Micro Optics Test Bench (MOTB) at the ID06 ESRF beamline at the energy of $12.9 \mathrm{keV}$. The CCD detector with a spatial resolution of about 0.9 $\mu \mathrm{m}$ was placed at a distance of $4.1 \mathrm{~m}$ from the bilens. The boron fibre was placed in front of the interferometer and a set of interference fringe images were recorded when the sample moved across the optical axis. Cross-sections of interference fringes for each fibre position were obtained and collected in an experimental scan image, which is shown in Fig. 1b. The shifts of interference fringes appearing during scanning are clearly demonstrated. The analysis of this scan image allows to reconstruct the phase (Fig 1c.) and absorption profiles of the sample. These results are consistent with the calculated phase and absorption profiles of the boron fibre. 
Knowing that the bilens system generates two diffraction limited (up to tens of $\mathrm{nm}$ ) focal spots with a separation of several tens of microns, the sample can be placed in the focal plane of the lenses. In this case, the small size of the focused beams provides extremely high phase and spatial resolutions.

In the case of an interferometer consisting of a multiple set of parallel arrays of identical planar refractive lenses [4], the created interference field is similar to one produced by grating interferometers and can be described by the Talbot imaging formalism. Unlike grating interferometers, a multilens interferometer can be used in a hard X-ray region $(>30 \mathrm{keV})$, while the manufacturing of a thick micron-pitch grating interferometer is problematic. Similarly to the bilens interferometer, the sample can be located in front and after the interferometer.

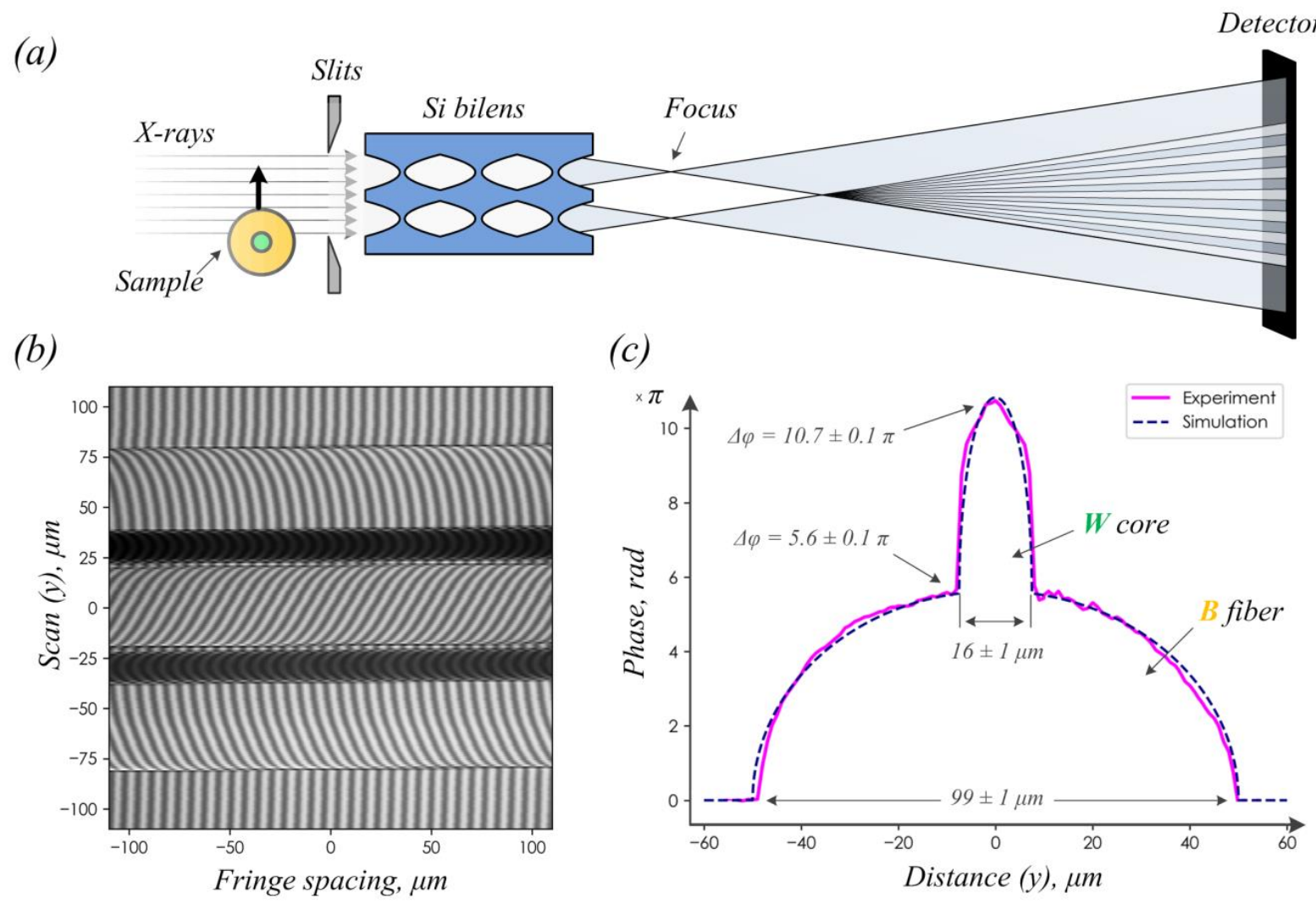

Figure. 1. (a) Experiment layout for phase contrast imaging technique using bilens. (b) Experiment scan and (c) the reconstruction of the phase profile of the boron fiber.

The proposed phase contrast imaging technique will allow studying natural and advanced man-made nanoscale materials, such as self-organized biological systems, photonic crystals and nanoelectronics [5].

References:

[1] A. Momose, et al., Japanese journal of Applied Physics, 42 (2003) L866.

[2] C. David, et al, Appl. Phys. Lett. 81 (2002) 3287.

[3] A. Snigirev, et al, Phys. Rev. Lett. 103 (2009) 064801.

[4] A. Snigirev, et al, Optics express 22 (2014) 25842-25852.

[5] The authors acknowledge funding from the Ministry of Education and Science of the Russian Federation grant contract № 14.Y26.31.0002. 